\title{
Determination of the Association of Urine Prostate Specific Antigen Levels with Anthropometric Variables in Children Aged 5-14 Years
}

\author{
Ioannis Efthimiou, Georgios Ferentinos, Georgios Tsachouridis, Girish Sardana, Eleftherios \\ Diamandis
}

Department of Urology (IE), University Hospital of Alexandroupolis, Alexandroupolis, Greece, Hellenic Centre for Diseases Control and Prevention (GF), Athens, Greece, Department of Pediatric Urology (GT), Children's Hospital "Agia Sofia”, Athens, Greece and Department of Pathology and Laboratory Medicine (GS, ED), Mount Sinai Hospital, Toronto, Canada.

\begin{abstract}
Purpose: Calculation of PSA is possible in human fluids even if it presents in very low concentrations with the help of hypersensitive immunodiagnostic methods. The periurethral glands represent one of the potential sources of urine prostate specific antigen (uPSA) in both sexes but the purpose of studying PSA levels in children is still unclear in the literature. In this pilot study we studied uPSA in a small cohort of normal, pre and post pubertal children, in relation to standard anthropometric variables.

Materials and Methods: The study cohort consisted of 58 children 5-14 years old (42 boys/16 girls). Height, weight, body mass index (BMI) and the respective stature-for-age, weight-for-age and BMI-for-age percentiles of the sample were determined. uPSA levels were measured using a third generation immunodiagnostic method (DPC Immulite ${ }^{\circledR}$ ) that has a lower limit of detection of $3 \mathrm{ng} / \mathrm{L}$. When levels of PSA were above the upper limit of detection, uPSA levels were assessed using the ROCHE technique.

Results: uPSA levels tend to be higher in male than female children ( $\mathrm{p}=0.091$, linear regression analysis). uPSA was measurable only in 3/16 girls (18.75\%). Measurable uPSA was found in 18/42 boys (42.8\%). The range of urine PSA in boys was $0-161000 \mathrm{ng} / \mathrm{L}$ (mean $10561.9 \pm 31830.48 \mathrm{ng} / \mathrm{L}$ ). Statistical analysis with linear regression showed correlation with height and age in boys.

Conclusions: The use of hypersensitive assays allows calculation of uPSA in childhood. The values of this variable are measurable in both sexes and related with gender. In boys, uPSA was correlated with age and height but not with other variables tested. Further studies are required to clarify this field.
\end{abstract}

Key words: prostate-specific antigen; child; urine

Int Braz J Urol. 2010; 36: 202-8

\section{INTRODUCTION}

Prostate specific antigen (PSA) was discovered initially in seminal fluid as $\gamma$-seminoglobulin in the late 1960s (1). Since then there has been a great number of discoveries and developments in the field of this marker. It is now clear that PSA is not tissue or gender specific. Studies have shown its presence in 
other tissues and especially in female tissues, such as periurethral glands, breast, endometrium and ovarian tissue $(2,3)$.

Advances in clinical biochemistry in the last decade have made possible measurement of PSA using highly sensitive assays in body fluids with extremely low concentrations. One of these methods, the third generation DPC Immulite ${ }^{\circledR}$ method (Diagnostics Products Corp., Los Angeles, CA) has a detection limit of $3 \mathrm{ng} / \mathrm{L}$ and a calibration range up to 20000 $\mathrm{ng} / \mathrm{L}$ (4).

Previous studies have shown that serum PSA can be calculated not only in boys in the different stages of puberty but also in girls, although in the latter it is encountered in lower concentrations and with lower frequency $(5,6)$. Recently PSA was measured in the urine of children of both sexes (7). The role of uPSA in children has not been investigated yet. Prostate development in childhood is related with testosterone activity, androgen receptor and 5-alpha reductase activity in the target organ (8-10). Diseases that affect the levels and activity of the above substances such as disorders of sexual differentiation and hypospadias (11) could potentially decrease PSA production in urine. uPSA could be used as a non-invasive marker for these diseases.

In the present study, we collected urine sample from healthy boys and girls aged 5-14 years, calculated uPSA levels and investigated the association of PSA with standard anthropometric variables.

\section{MATERIALS AND METHODS}

We studied 58 healthy children (42 boys and 16 girls) aged 5-14 years, not displaying any endocrine, neurological, nephrological or urogenital disorders. Children taking any kind of medication or with a fever at the time of collection of urine specimen were excluded from the study. Samples were collected from children who visited the outpatient department for minor health problems and from normal children of the hospital staff. Subjects were natives of Greece. This research study was approved by the Research Ethics Committee and local school authorities.

Each sample was collected according to the following protocol: Written consent was first ob- tained from the parents of each child who were fully informed about the study design. Then the first 5 $\mathrm{mL}$ of urine was collected from the first morning urination between 7.00-9.00 hours. The sample was centrifuged at $3000 \mathrm{~s} / \mathrm{min}$ for 2 minutes and the top $2 \mathrm{~mL}$ of supernatant was stored at $-30^{\circ} \mathrm{C}$. In addition, height and body weight was recorded in order to calculate the respective stature-for-age, weight-forage and body mass index (BMI)-for-age percentiles of the sample. uPSA was calculated using the DPC Immulite method, which has a lower limit of detection of $0.003 \mu \mathrm{g} / \mathrm{L}$. Samples that had PSA levels outside of the measurable range of the DPC Immulite were measured using the ROCHE technique. Biostatistical analysis was performed using the Stata 9.0 software suite. We used the Pearson correlation to study correlations between continuous variables, the ANOVA or linear regression analysis to study continuous over categorical variables and Pearson's chi square test was utilized to study categorical variables. For all statistical tests a significance level of 0.05 was used. As far as the age of the subjects was concerned, we examined it not only as a continuous variable, but also as a binomial categorical variable as well in two groups: a childhood group (26 boys, $\leq 12$ years old) and adolescent group ( 16 boys, $>12$ years old).

\section{RESULTS}

PSA was detected in the urine of 18 of 42 boys $(42.8 \%)$ and in three of 16 girls $(18.75 \%)$. uPSA levels tend to be higher in male than female children $(p=0.091$, linear regression analysis). Two girls 6 and 11 years old respectively had a uPSA value of $10 \mathrm{ng} / \mathrm{L}$ and another one 13.5 years old had uPSA of $316 \mathrm{ng} / \mathrm{L}$. The range of uPSA in boys presented greater variability. Especially in boys 5-8 years old, uPSA activity was detected in 5 of 14 specimens (35.7\%). In 4 cases the range was 3-10 $\mathrm{ng} / \mathrm{L}$ and in one case the activity was $<3 \mathrm{ng} / \mathrm{L}$. In the group of boys aged $8-12$ years old it was detected in $2 / 12$ (16.6\%) specimens with values 20 and $79 \mathrm{ng} / \mathrm{L}$. In boys older than 12 years, uPSA was detected in $11 / 16(68.75 \%)$ specimens with a range of 200 $\mathrm{ng} / \mathrm{L}-161000 \mathrm{ng} / \mathrm{L}$. Statistical analysis of uPSA in 
boys showed a moderate strong positive relation with age (p: 0.043, rho: 0.317) and height (p: 0.043, rho: 0.318 ). The relationship of uPSA to age and height is shown Figures-1 and 2 respectively. Pearson's correlation coefficients for uPSA showed no correlation with weight, BMI, stature-for-age, weight-

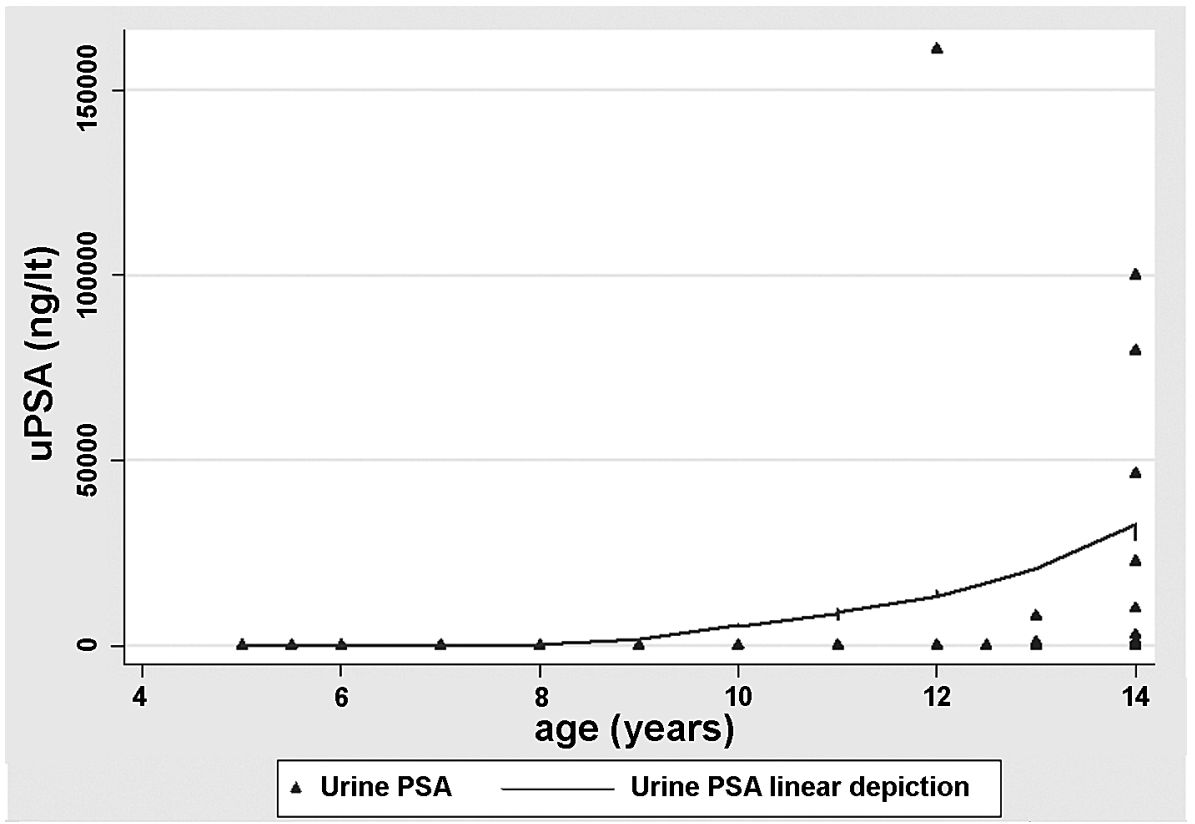

Figure 1 - Urine PSA in boys in relation to age. There is a sharply increase of uPSA in boys after the age of 12 years, when they come into adolescence.

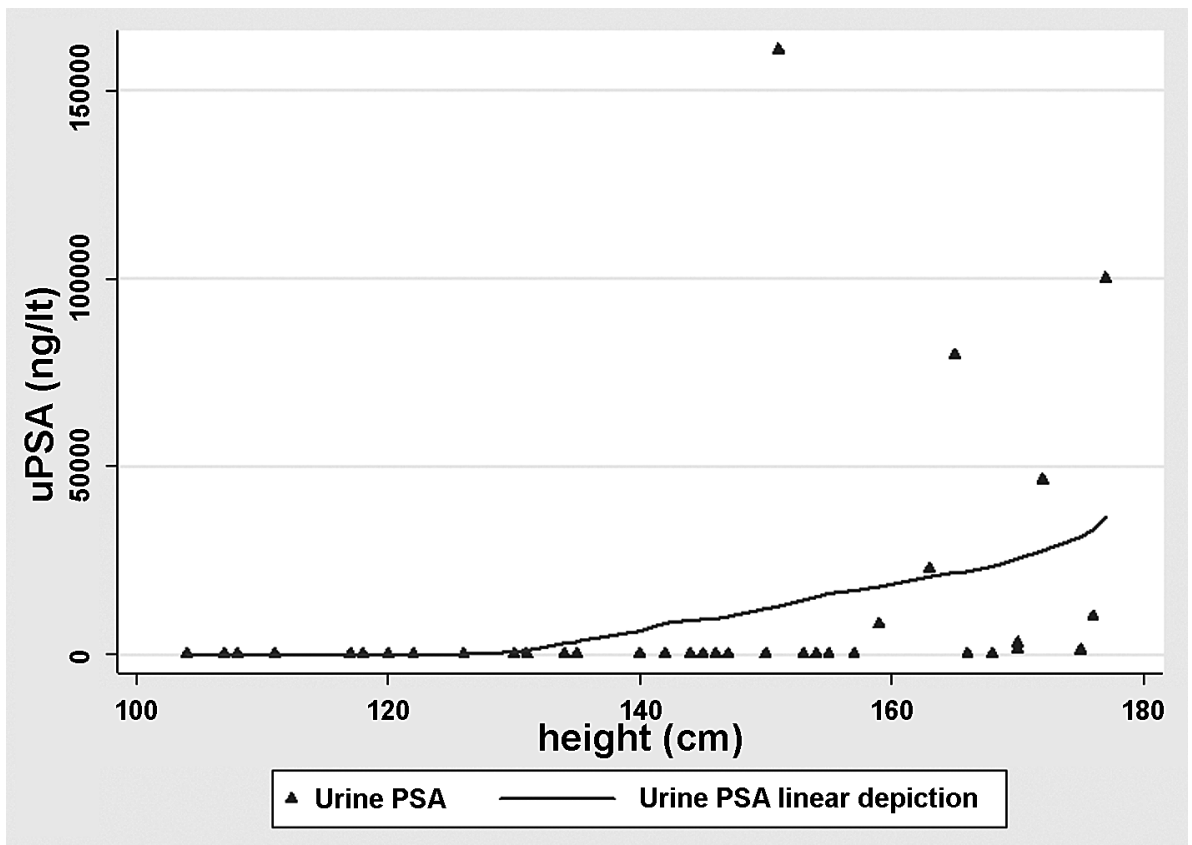

Figure 2 - Urine PSA in boys in relation to height. The slope is proportional to that of Figure-1 reflecting as a major contributor of increase entry of boys into adolescence. 
Table 1 - Results of statistical analysis in boys.

\begin{tabular}{lcc}
\hline Variable & rho & p Value * \\
\hline Age & 0.316 & 0.043 \\
Weight & 0.225 & 0.156 \\
Weight-for-age & -0.046 & 0.773 \\
Height & 0.318 & 0.042 \\
Height-for-age & 0.142 & 0.376 \\
BMI & 0.014 & 0.93 \\
BMI-for-age-percentile & -0.214 & 0.179 \\
\hline
\end{tabular}

* For all statistical tests a significance level of 0.05 was used. $B M I=$ mass body weight.

for-age or BMI-for-age percentiles (Table-1). Boys were divided according to their age into two groups: childhood ( 26 boys, $\leq 12$ years old) and adolescent group ( 16 boys, $>12$ years old) in order to study if there was any relation of weight to uPSA. Sub-classification of each group according to BMI-for-age percentiles in normal, overweight and obese was also performed. We also investigated whether BMI-for-age percentiles classification correlates with age group using Pearson's chi square. No relation was found ( $p$ $=0.69$ ). Linear regression analysis did not show any relation of UPSA with obesity in neither childhood nor in adolescence groups.

\section{COMMENTS}

It is well known that serum PSA levels rise sharply with increasing stage after males reach puberty and this rise has been shown to correlate with testosterone levels $(6,12)$. A recent study from Antoniou et al. showed that serum PSA levels do not differ significantly between the two sexes until the age of 12 years, with median values of $38.41 \mathrm{ng} / \mathrm{L}$ (range: 1-2768) and 4.059 (1-287) ng/L for boys and girls respectively. After the age of 12, serum PSA levels in boys are seen to increase sharply (13). Another study by Sato et al. found that there is a gradual increase in uPSA activity from 1-4 month old infant males and boys older than 10 years of age. In boys 0.3-9 years there was no uPSA activity (7). These results reflect the previous study by Goldfarb et al. who showed that PSA levels in prostatic tissue were high at birth and decreased after 6 months, and subsequently reappeared by 10 years of age and increased during puberty (14).

In our study, we showed that uPSA was detectable in $26 \%$ of boys aged 5-12 years old within the range of 2-79 $\mathrm{ng} / \mathrm{L}$. In boys older that 12 years uPSA values increased up to $161000 \mathrm{ng} / \mathrm{L}$. There was a statistically significant correlation between uPSA levels and height. Taller boys had higher levels of uPSA. This correlation disappeared when it was investigated in relation to stature for age percentiles because this variable is corrected for age. Statistical analysis of uPSA levels in boys with weight, BMI and the respective stature-for-age, weight-for-age and BMI-for-age percentiles of the population did not reveal any correlation.

uPSA was found only in a small percentage of girls in our sample (18.75\% of girls). Other studies have shown that PSA is detectable in $11-38 \%$ of adult females with mean values of 290 and 3.720 $\mathrm{ng} / \mathrm{L}$ respectively $(15,16)$. Periurethral glands or the "female prostate" are considered to be homologous to the prostate gland as these organs have the same embryological origin $(17,18)$. Periurethral glands in females are considered to be the primary site of PSA secretion in urine and manifest active secretion in approximately $66.7-80 \%$ of women $(2,17,18)$. These glands do not undergo developmental changes from fetal to adult age (17). Another possible source of female adult urine PSA is the reactive metaplastic changes of cystitis cystica and cystitis glandularis that occur in adult women (19). The presence of PSA in females is under regulation of steroid hormones especially androgens and progestin. Our results support the concept that periurethral glands do not (if at all) influence significantly the serum PSA in both sexes (20).

uPSA could be potentially used as a non-invasive marker of sexual development and abnormalities in boys. However, there are major disadvantages for its use. It requires hypersensitive PSA assays that are not always available in the biochemistry department. In addition values are not standardized and may fluctuate in the same subject from one urine sample to another (21). 
Our study design has certain limitations as it has involved a relatively small cohort of children, a single urine specimen from each child and the Tanner stage of the individuals was not recorded. Better designed studies which consider the above limitations will help to better define uPSA levels and variation in this population.

\section{CONCLUSION}

The use of hypersensitive assays allows calculation of uPSA in childhood. The values of this variable are measurable in both sexes and related with gender. In boys, uPSA is correlated with age and height but not with other variables tested. Further studies are required to clarify this field.

\section{CONFLICT OF INTEREST}

None declared.

\section{REFERENCES}

1. Hara M, Koyanagi Y, Inoue T, Fukuyama T: Some physico-chemical characteristics of " $\mathrm{y}$-seminoprotein", an antigenic component specific for human seminal plasma. Forensic immunological study of body fluids and secretion. VII. Nihon Hoigaku Zasshi. 1971; 25: 322-4.

2. Pollen JJ, Dreilinger A: Immunohistochemical identification of prostatic acid phosphatase and prostate specific antigen in female periurethral glands. Urology. 1984; 23: 303-4.

3. Yu H, Diamandis EP, Levesque M, Giai M, Roagna R, Ponzone R, et al.: Prostate specific antigen in breast cancer, benign breast disease and normal breast tissue. Breast Cancer Res Treat. 1996; 40: 171-8.

4. Ward AM, Catto JW, Hamdy FC: Prostate specific antigen: biology, biochemistry and available commercial assays. Ann Clin Biochem. 2001; 38: 633-51.

5. Randell EW, Diamandis EP, Ellis G: Serum prostatespecific antigen measured in children from birth to age 18 years. Clin Chem. 1996; 42: 420-3.

6. Vieira JG, Nishida SK, Pereira AB, Arraes RF, Verreschi IT: Serum levels of prostate-specific antigen in normal boys throughout puberty. J Clin Endocrinol Metab. 1994; 78: 1185-7.

7. Sato I, Yoshikawa A, Fugimoto M, Shimizu K, Ishiwari A, Mukai T, et al.: Urinary prostate-specific antigen is a noninvasive indicator of sexual development in male children. J Androl. 2007; 28: 150-4; discussion 155-7.

8. Schuur ER, Henderson GA, Kmetec LA, Miller JD, Lamparski HG, Henderson DR: Prostate-specific antigen expression is regulated by an upstream enhancer. J Biol Chem. 1996; 271: 7043-51.

9. Lee C, Kozlowski M, Grayhack JT: Aetiology of benign prostatic hyperplasia. Urol Clin N Am. 1995; 22: 237-46.

10. Xue Y, Sonke G, Schoots C, Schalken J, Verhofstad A, de la Rosette J, et al.: Proliferative activity and branching morphogenesis in the human prostate: a closer look at pre- and postnatal prostate growth. Prostate. 2001; 49: 132-9.

11. Diamond DA. Sexual differentiation: Normal and abnormal. In: Wein AJ. Wein: Campbell-Walsh Urology. 9th (ed.), Saunders, Elsevier. 2007: pp. 3799.

12. Juul A, Müller J, Skakkebaek NE: Prostate specific antigen in boys with precocious puberty before and during gonadal suppression by GnRH agonist treatment. Eur J Endocrinol. 1997; 136: 401-5.

13. Antoniou A, Papanastasiou P, Stephanidis A, Diamandis E, Androulakakis PA: Assessment of serum prostate specific antigen in childhood. BJU Int. 2004; 93: 838-40.

14. Goldfarb DA, Stein BS, Shamszadeh M, Petersen RO: Age-related changes in tissue levels of prostatic acid phosphatase and prostate specific antigen. J Urol. 1986; 136: 1266-9.

15. Schmidt S, Franke M, Lehmann J, Loch T, Stöckle M, Weichert-Jacobsen K: Prostate-specific antigen in female urine: a prospective study involving 217 women. Urology. 2001; 57: 717-20.

16. Breul J, Pickl U, Hartung R: Prostate-specific antigen in urine. Eur Urol. 1994; 26: 18-21.

17. Wernert N, Albrech M, Sesterhenn I, Goebbels R, Bonkhoff H, Seitz G, et al.: The 'female prostate': location, morphology, immunohistochemical characteristics and significance. Eur Urol. 1992; 22: 64-9.

18. Tepper SL, Jagirdar J, Heath D, Geller SA: Homology between the female paraurethral (Skene's) glands and the prostate. Immunohistochemical demonstration. Arch Pathol Lab Med. 1984; 108: 423-5.

19. Nowels K, Kent E, Rinsho K, Oyasu R: Prostate specific antigen and acid phosphatase-reactive cells 
in cystitis cystica and glandularis. Arch Pathol Lab Med. 1988; 112: 734-7.

20. Diamandis EP, Yu H: Nonprostatic sources of prostate-specific antigen. Urol Clin North Am. 1997; 24: $275-82$.
21. Takayama TK, Vessella RL, Brawer MK, True LD, Noteboom J, Lange PH: Urinary prostate specific antigen levels after radical prostatectomy. J Urol. 1994; 151: 82-7.
Accepted after revision:

October 10, 2009

\section{Correspondence address:}

Dr. Efthimiou Ioannis

Platonos 58 TK 12132

Athens, Greece

Fax number: + 3022 7135-0236

E-mail: efthimiou ioannis@hotmail.com

\section{EDITORIAL COMMENT}

My concerns regarding the paper are more related to the limited relevance of the issue in clinical practice in pediatric urology. Despite the arguments of the authors that "-uPSA has an importance as a potentially noninvasive marker (in contrast with serum hormones like FSH and testosterone) which could be used easier in that part of the population" the first task of the authors would be to define the sensitivity of the method, which was very poor in the series ( $26 \%$ boys $5-12$ years and $18.75 \%$ of girls).

Dr. Antonio Macedo Jr. Federal University of Sao Paulo Sao Paulo, SP, Brazil E-mail:amcdjr@uol.com.br

\section{EDITORIAL COMMENT}

In this straightforward work, the authors evaluate the value of urinary PSA in children of both sexes aged 5-14 years. They found that urinary PSA (uPSA) values differ significantly between boys and girls, being measurable in only $18.75 \%$ of girls, against $42.8 \%$ of boys. Also they observed that in boys the uPSA values increased with age and height, but not with weight (obesity).
Although somewhat expected, this information is quite interesting, since in a previous work (ref. 13), the same group showed that the serum PSA(sPSA) does not differ significantly between boys and girls until 12 years of age (difference being observed in boys with the increase of sPSA due to prostatic development during puberty). Furthermore, another study (ref. 7) showed no detection of UPSA in children aged 0.3 to 9 years. 
The simultaneous evaluation of both uPSA and sPSA, with the same hypersensitive assay, would certainly clarify these contradictory data and enhance the information presented in this work.

One also wonders why only the first $5 \mathrm{~mL}$ of the first morning urination were collected for analysis, since a midstream sample seems to more appropriate for biochemical evaluation.
Although easier to collect than blood samples, uPSA still seems to be less reliable for evaluation than sPSA in this age group, therefore its practical value is still to be determined.

\author{
Dr. Francisco T. Denes \\ Division of Urology \\ University of Sao Paulo, USP \\ Sao Paulo, Brazil \\ E-mail:f.c.denes@br2001.com.br
}

\section{REPLY BY THE AUTHORS}

We agree with the comments of Dr. Macedo and we feel that a larger and better designed study would solve the problem of low sensitivity.

We also agree with the comments of Dr. Denes and would like to emphasize that although in our previous work sPSA was measurable in both sexes in childhood (1); in our last work, uPSA was measurable in $42.8 \%$ of males and in only $18.75 \%$ of female children. One could conclude that peri-urethral glands do not influence significantly the serum PSA in both sexes. Furthermore, Sato et al. (2) did not find a measurable uPSA in children form 0.3 to 9 years old. The above studies have many methodological differences (method of statistical analysis, assay, sample size, etc) that could explain the divergence in their results. We are in agreement with the suggestion that simultaneous evaluation of both urine and serum PSA in the same sample population using the same hypersensitive assay would further clarify the subject.

Regarding the relation of height and UPSA we should emphasize that this moderate positive relation (p: 0.043, rho: 0.318 ) was lost when the comparison was conducted between stature-for-age and uPSA. This practically means that we should be rather cautious to draw a definite conclusion between the two variables, considering that comparison of uPSA with stature-for-age is more accurate when referring in children population.

Regarding the sampling method of urine collection in our protocol, the first voided urine sample better reflects local PSA production by the prostate and urethra than the midstream sample (3).

\section{REFERENCES}

1. Antoniou A, Papanastasiou P, Stephanidis A, Dia $\neg$ mandis E, Androulakakis PA: Assessment of serum prostate specific antigen in childhood. BJU Int. 2004; 93: 838-40

2. Sato I, Yoshikawa A, Fugimoto M, Shimizu K, Ishiwari A, Mukai T, et al.: Urinary prostate-specific antigen is a noninvasive indicator of sexual development in male children. J Androl. 2007; 28: 150-4; discussion 155-7.

3. Iwakiri J, Granbois K, Wehner N, Graves HC, Stamey T. An analysis of urinary prostate specific antigen before and after radical prostatectomy: evidence for secretion of prostate specific antigen by the periurethral glands. J Urol. 1993;149:783-6. 